\title{
照Digital Publisher
}

ISSN 2588-0705

\section{Análisis de la situación económica de la producción de café en el cantón Zaruma en el periodo 2017 - 2020}

\section{Analysis of the economic situation of coffee production in the Zaruma canton in the period 2017 - 2020}

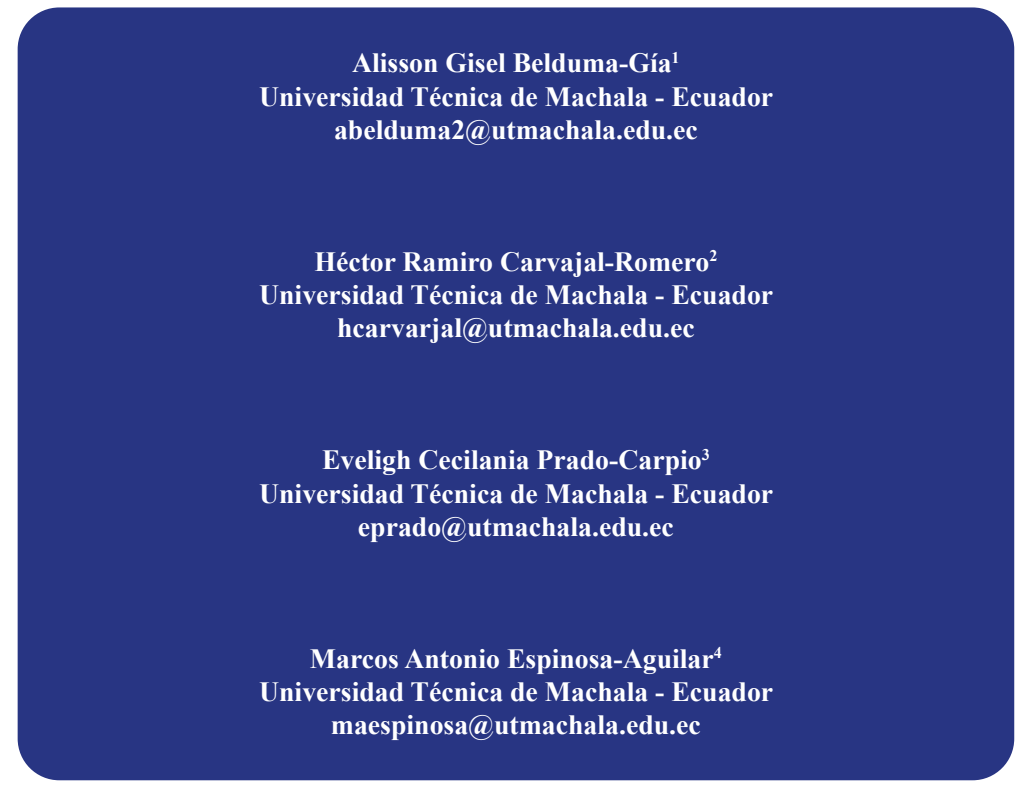

doi.org/10.33386/593dp.2022.2.1047

V7-N2 (mar-abr) 2022, pp. 229-238 | Recibido: 07 de febrero de 2022 - Aceptado: 14 de febrero de 2022 (2 ronda rev.)

\section{Estudiante de la carrera de Economía agropecuaria}

ORCID: https://orcid.org/0000-0002-1735-9322

2 Ingeniero Comercial Magister en Administración de Empresas. Docente de la Universidad Técnica de Machala ORCID: https://orcid.org/0000-0001-6303-6

3 Master de docencia e investigación por la Universidad Técnica de Machala, Ecuador. Dra. en Ciencias Agrarias, por la Universidad de Zulia, obteniendo Mención de honor en el Ph.D

ORCID: https://orcid.org/0000-0002-0225-5264

4 Master universitario en Iniciativa Empresarial: Análisis y Estrategias, 2019. Doctorando en Ciencias Agrarias y Ambientales ORCID: https://orcid.org/0000-0003-2608-0769 
Belduma-Gía, A., Carvajal-Romero, H., Prado-Carpio, E., \& Espinosa-Aguilar, M., (2022). Análisis de la situación económica de la producción de café en el cantón Zaruma en el periodo 2017 - 2020. 593 Digital Publisher CEIT, 7(2), 229-238 https://doi. org/10.33386/593dp.2022.2.1047

Descargar para Mendeley y Zotero

\section{RESUMEN}

La presente investigación aborda el análisis de la situación económica de los productores de café en el cantón Zaruma, Provincia de El Oro - Ecuador durante el periodo 2017-2020. El alcance de la presente investigación es de tipo descriptivo, la herramienta utilizada para la obtención de información primaria fue una encuesta con una muestra de 50 productores de café, el cual permite identificar los factores que inciden en el ingreso de los productores de café en el cantón Zaruma, que en los últimos años ha dejado de ser rentable y se propone una nueva forma de entender el papel de sector cafetero en el desarrollo económico local. Por lo tanto, se concluye que es de vital importancia de la reorganización del sector cafetero para que los productores sean más competitivos en el mercado internacional. Las tres cuartas partes de los productores de café en el cantón Zaruma oscilan en un rango de 55 a 60 años, además las unidades productivas están lideradas en un $70 \%$ por el género masculino. Más de la mitad de las unidades productivas de café están en manos de pequeños productores con rango de 1 a 5 ha. Dado este contexto el $44 \%$ de los productores venden a $\$ 250$ dólares el quintal de café, logrando obtener ingreso promedio mayor a $\$ 1500$ por hectárea al año, alcanzando beneficios potenciales para los productores que adoptaron estándares de sustentabilidad, incluyendo mejores condiciones de comercio, así como el valor de preservar el medio ambiente.

Palabras claves: demanda; consumo; producción; exportación; comercialización

\section{ABSTRACT}

The present research addresses the analysis of the economic situation of coffee producers in the Zaruma canton, El Oro Province - Ecuador during the period 2017-2020. The scope of this research is descriptive, the tool used to obtain primary information was a survey with a sample of 50 coffee producers, which allows identifying the factors that affect the income of coffee producers in the Zaruma canton, which in recent years has ceased to be profitable and proposes a new way of understanding the role of the coffee sector in local economic development. Therefore, it is concluded that it is of vital importance to reorganize the coffee sector for producers to be more competitive in the international market. Three quarters of the coffee producers in the canton of Zaruma are between 55 and 60 years of age, and 70\% of the productive units are led by men. More than half of the coffee production units are in the hands of small producers with a range of 1 to 5 ha. Given this context, $44 \%$ of the producers sell at $\$ 250$ dollars per quintal of coffee, obtaining an average income of more than $\$ 1500$ per hectare per year, reaching potential benefits for producers who adopted sustainability standards, including better trading conditions, as well as the value of preserving the environment.

Keywords: demand; consumption; production; export; marketing 


\section{Introducción}

El café (Coffea arabica L.) se cultiva en más de 56 países del mundo, siendo uno de los principales productos agrícolas de producción en África, América Latina y Asia. Su alto valor económico, su importancia ambiental y su valor social, han hecho de la producción de café una actividad agrícola trascendental en las economías locales, ya que su producción involucra las familias campesinas de las zonas rurales (Chango, 2021).

Durante las últimas dos décadas se han presentado grandes transformaciones en el mercado cafetalero como consecuencia de la desregulación del mercado (liberación de aranceles principalmente a los países africanos) lo cual provoco una mayor competencia y variación de productos.

Por lo tanto, se debe estudiar cómo se asignan esos recursos, de forma eficiente para satisfacer esas necesidades. Evidentemente, si queremos una asignación "eficiente" de dichos recursos, tendremos que tener en cuenta que no se puede mejorar el consumo de un recurso, sin empeorar el consumo del otro. A su vez, el análisis económico presenta dos enfoques de estudio: La Microeconomía, y la Macroeconomía en los procesos de la producción agrícola(Vaca et al., 2018).

En teoría el análisis económico no es mas allá que una base del estudio teórico y académicos sobre la economía. Su principal objetivo es el estudio del comportamiento de los individuos y organizaciones en la toma de decisiones para obtención de mejores resultados ya sea en empresas administrativa, campo agropecuario ya que la economía se encuentra en todos lados. (Márquez et al., 2019).

La economía de un país depende en gran medida del desarrollo del sector productivo en el que las empresas juegan un papel relevante, de su desempeño depende que se genere crecimiento o estancamiento. Por tal razón la productividad y competitividad es el producto de las formas de realizar el trabajo, la creatividad e ingenio para formular estrategias que permitan a las organizaciones optimizar los recursos, mejorar los costos y posicionarse en el mercado (García \& Quezada, 2021).

Ecuador aparece a nivel mundial como productor, exportador y consumidor de café, su cultivo está distribuido en las cuatro regiones geográficas del país, en su mayoría se trata de pequeñas unidades de producción y sistemas agroforestales, por lo cual constituye un beneficio para el ambiente, además de esto, tiene importancia económica por tratarse de una fuente de ingresos, y social porque en las cadenas de producción del café participan muchas etnias y pueblos (Ponce, et al., 2018). Para el año 2018 el país contaba con 61254 ha de café plantadas, de las cuales se obtuvieron 28542 toneladas de producción, equivalente a 0,47 ton h-1 (Pincay \& Unda, 2020).

Dado estos factores la producción de café en el Ecuador se ubica como uno de los mejores productores en América del Sur; este sector productivo involucra en la generación de empleo a 200.000 ecuatorianos, y aporta al $3 \%$ al Producto Interno Bruto.

El 2020 Ecuador recibió \$77,8 millones por exportaciones de café, lo más bajo desde el 2005 , cuando fueron $\$ 88,4$ millones, en los últimos siete años la reducción es muy drástica: $68 \%$ menos en volumen y $72 \%$ en ingreso de divisas para el país (Asociación Nacional de Exportadores de Café, 2020).

Para los ecuatorianos, tiene importancia en los órdenes: económico, social, ambiental, y salud humana. En lo económico, constituye una fuente de divisas e ingresos para los actores de las cadenas del café, que en el 2020 representó ingresos por USD 145.354.370,31. En lo social, en las cadenas del café se involucran muchas etnias y pueblos en 23 de las 24 provincias de Ecuador, dispersos en un amplio tejido social. En lo ambiental, se cultiva básicamente en sistemas agroforestales. En lo referente a la salud humana, el consumo de café muestra correlación inversa con el riesgo de diabetes tipo 2, daño hepático $\mathrm{y}$ enfermedades neurodegenerativas como el 
Parkinson (Ponce et al., 2018).

La dinámica de la oferta y demanda, así como el comercio internacional de café hasta 1989 era controlada mediante una serie de Acuerdos Internacionales de café, cuyo objetivo era de conservar precios estables y administrar la oferta de dicho bien. Desde 1990 el mercado de café ha estado sujeto a un mercado libre de oferta y demanda. En el período que este mercado libre ha estado vigente se han presentado dos periodos de precios bajos (1989-1993 y 1999-2004), siendo el de 1999-2004 conocido como la crisis del café. De acuerdo con récords históricos de precios posteriores al fin de esta crisis (2004), éstos han mejorado en relación con los precios durante las dos crisis antes mencionadas, pero la ICC reporta alta variabilidad en los precios debido al incremento de costos de los insumos para la producción de café. (Figueroa Hernandez, Perez Soto, Godinez Montoya, \& Perez Figueroa, 2018)

De acuerdo al reporte de coyuntura del (Banco Central del Ecuador, 2019), la producción de café, tiene resultados positivos dando un crecimiento del $12 \%$, pasa 905 ton producidas en el año 2016 a 5065 ton en 2018, en un área menor. Aumento en la producción que está relacionado a la siembra de nuevos cafetales de café arábigo por medio de un convenio firmado entre los productores, el Ministerio de Agricultura y ganadería (MAG) y el Banco Central del Ecuador. Durante el año 2018 la producción de café en la provincia de El Oro fue de 133 ha plantadas, de las cuales se obtuvo 25 ton equivalente a 0,20 ton h-1 (Instituto Nacional de Estadística y Censos, 2019)

Ecuador tiene entre de sus productos estrella al café, en iguales condiciones al banano, cacao y al camarón, produciéndose en sectores tradicionales de la costa, pero existe un pequeño rincón en las montañas ecuatorianas donde, gracias a las características del lugar, se produce un café de una calidad muy superior, con sabores y texturas inigualables, lamentablemente este producto no se ha exportado todavía, perdiendo grandes cantidades de dinero y fama mundial. Este lugar tiene el nombre de Zaruma, conocida como la sultana de El Oro. (Bello, 2016)

La región alta de la provincia de El Oro, en donde se encuentra el cantón Zaruma, es un área propicia para el sembrío de café arábigo de calidad Premium debido a que sus tierras se encuentran en una altura de entre 900 a 2000 metros sobre el nivel del mar, su clima agradable y la fertilidad del campo son otros factores que aportan a las privilegiadas características que tiene el café producido en estas tierras. (Bello, 2016)

En el cantón se cultivan las dos principales especies comerciales en el contexto mundial, Coffea arábica L (café arábico) y Coffea canephora Pierre ex Froehner (robusta). Las tecnologías de producción generadas y validadas han permitido elevar la productividad con fincas de alto rendimiento. Sin embargo, el mayor problema que enfrenta la caficultura zarumeña son los bajos niveles de producción, con un promedio de apenas $270 \mathrm{~kg} / \mathrm{has}$ (Pincay \& Unda, 2020)

En el sector la cobertura agropecuaria en las zonas de pequeñas parcelas de Sierra ( $\leq 5$ ha) cubre una superficie aproximada de 9.220 ha, que es representada por pastizales y sembríos de cañas de azúcar. Como parcelas de la región costa se encuentra algunos productos agropecuarios como: la naranja, caña de azúcar, mandarina, maíz duro, cacao, yuca y café.

El café es un producto muy comercializado en este cantón, ya que por su agradable aroma es muy apetecido por las personas del sector o de cantones cercanos; a su vez es comercializado en otros mercados y tiene gran acogida por la mayoría de las personas.

El cantón Zaruma se caracteriza por una pequeña agricultura organizada en la forma de producción campesina es definida por dos características esenciales, el trabajo agrícola, el cual proviene de la propia familia, y el tamaño de la tierra, que impiden al grupo familiar campesino obtener un nivel de ingreso superior al de subsistencia, dado aquello la economía del cantón su fortaleza es la minería, las divisas que 
se generan permiten el desarrollo económico y social.

El café es un cultivo que creció de manera importante de a lo largo de las décadas en Zaruma, siendo incluso una alternativa mas reciente por localidades vecinas, para sustituir la producción de otros tipos de cultivos o actividades pecuarias. Por lo descrito la presente investigación tiene como objetivo, analizar la situación económica de la producción de café en el cantón Zaruma durante el periodo 2017-2020, con la finalidad de identificar los factores que inciden en el ingreso de los productores.

\section{Materiales y métodos}

El presente estudio se desarrollará en la provincia de El Oro, centrándose en el cantón Zaruma; el cantón seleccionado está ubicado en la parte alta de la provincia de El Oro, su temperatura varía de $15{ }^{\circ} \mathrm{C}$ a $26^{\circ} \mathrm{C}$, en raras ocasiones baja a menos de $13{ }^{\circ} \mathrm{C}$ o sube a más de $28{ }^{\circ} \mathrm{C}$, sus coordenadas geográficas son: latitud: $3^{\circ} 41^{\prime} 0^{\prime} \mathrm{S}$, Longitud: $79^{\circ} 36^{\prime} 0$ " O, altitud desde 500 a $3500 \mathrm{msnm}$.

El alcance de la presente investigación es de tipo descriptivo, la herramienta utilizada para la obtención de información primaria fue una encuesta directa como lo recomienda (López et al., 2017) para estudios descriptivos relacionados aspectos socioeconómicos. En el cantón Zaruma existen un total de 50 unidades productivas de café, sin embargo, no será necesaria la aplicación de la fórmula de la muestra, considerando que se tomará el total existente de productores de café. Para la recolección de información primaria se realizó una encuesta, que estuvo estructurada por 6 preguntas, que fueron estructuradas tres para el análisis socioeconómico, y tres para el aspecto de productividad (Valdez \& Vizcaya, 2009).

Identificado y clasificado la información necesaria que se requiere, realizar el analisis de la producción de café en cantón Zaruma en el periodo 2017-2020. El trabajo investigativo está basado en diversos documentos como revistas científicas, plataformas agroeconómicas, Banco Central del Ecuador y Ministerio de Agricultura y Ganadería (MAG) relacionados con el tema de la producción de café, con enfoque macro y micro.

Para el desarrollo de la investigación se realizaron las siguientes actividades:

1.- Revisión bibliográfica de los diferentes aportes relacionados al contexto de la investigación.

2.- Análisis de la producción de café en el cantón Zaruma, hectáreas producidas, aporte al PIB Nacional y consumo Per cápita de café en el Ecuador, el cual se lo realizó a través de la revisión de información secundaria mediante la (Web del Banco Central del Ecuador), Pro Ecuador.

3.- Importancia de la producción cafetera en la economía local.

La informacion recolectada fue tabulada en excel 2016 complementando con la distribución de frecuencias absolutas, que representan los porcentajes de casos en cada categoría, por lo tanto, se acumuló los porcentajes para determinar la incidencia del estudio realizado. Posteriormente se procesó en el software estadístico SPSS Versión 24.0, el cual permite realizar el análisis descriptivo mediante el uso de figuras y tablas para su respectiva interpretación de resultados de la investigación.

\section{Resultado y discusiones}

El café (Coffea arábica L.) se cultiva en diferentes países del mundo por alto valor económico, su importancia ambiental y su valor social, han hecho de la producción de café una actividad agrícola muy importante para la economía en desarrollo, ya que en sus procesos de producción están involucradas principalmente familias campesinas.

Las tres cuartas partes de los productores de café en el cantón Zaruma oscilan en un rango de 55 a 60 años de edad, además las unidades productivas están lideradas en un $70 \%$ por los jefes de hogar, mientras que el 30\% de mujeres son las jefas de hogar como se observa en la 
Figura

\section{Figura 1}

Demografía del cantón Zaruma de productores de café. Distribución por género y rango de edades

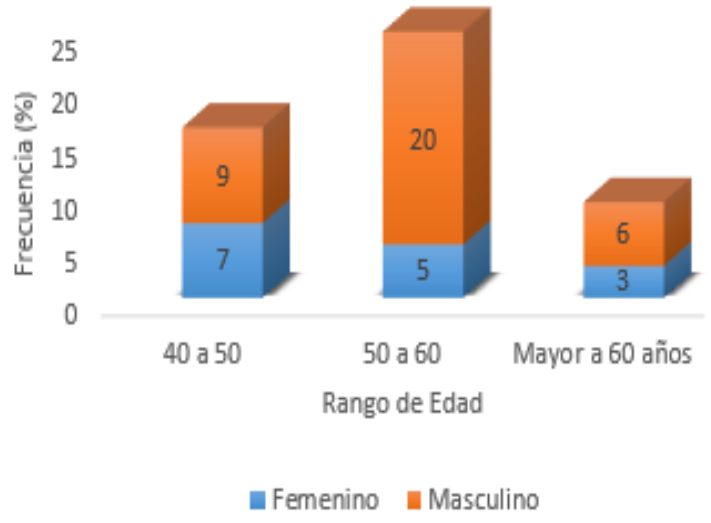

Los resultados obtenidos en las encuestas señalan el $44 \%$ de los productores de café poseen 1 a 5 ha que están dentro del segmento de pequeños productores, mientras que el 30 $\%$ de estas familias caficultoras poseen de 5 a 10 ha, el cual les permite suplir las necesidades básicas de subsistencia, por otra parte del sector productor de café contienen grandes productores que representan el $26 \%$ en términos de superficie cultivada Figura 2, a la vez, a este cantón corresponden los mejores rendimientos en la cosecha, y se ubica como el mayor productor de café de la Provincia de El Oro.

Datos que coinciden en un estudio realizado por Figueroa et al. (2019), que caracteriza que el tamaño que definen una agricultura de las tierras del campesinado son fáciles de establecer, pero en la mayoría de los casos, estos tamaños parecen corresponder a 5 o 10 ha de tierra, dependiendo de cada localidad. Además en varias investigaciones realizadas han concluido que el $25 \%$ de la población total de las regiones suramericanas se compone de familias campesinas.
Figura 2

Cantidad de hectáreas destinadas a la producción de café

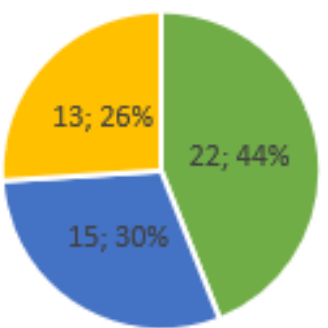

- a) 1 a $5 \mathrm{Ha} \quad$-b) 5 a $10 \mathrm{ha} \quad$ "c) Mayor a $10 \mathrm{Ha}$

Con la información recolectada en campo sobre la producción promedio de café en el cantón Zaruma durante el periodo 20172020 , se obtuvieron los siguientes datos el $50 \%$ de los productores obtienen un rendimiento de 20 a 30 quintales por ha, el $24 \%$ producen de 10 a 20 quintales por ha, mientras que el $26 \%$ de los productores dedicados a esta rama producen de 5 a 10 quintales por ha. En relación con los demás cantones productores de café, el cantón Zaruma lidera en rendimiento por ha de café Figura 3. Para alcanzar estos óptimos niveles de producción se han implementado algunas estrategias y medidas; como procesos de control de la producción, el cuidado de la calidad como un acuerdo entre los productores, de tal forma que sólo se comercialice un producto de calidad, con el objeto de percibir mejores ingresos. 


\section{Figura 3}

Producción promedio de café por ha periodo 2017-2020

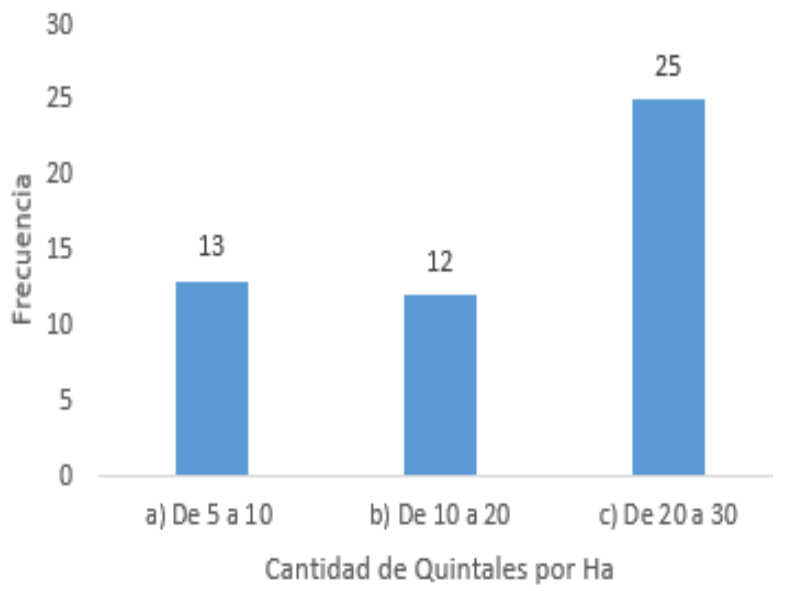

Los precios del café en la provincia de El Oro varían de acuerdo a diferentes factores entre ellos el tipo de café, la calidad del producto, el cantón Zaruma se ha caracterizado por ser uno de los mayores productores de café, el precio por quintal de mayor valor se encuentra a $\$ 250,00$ que representa el $44 \%$ de los productores lo comercializan a este precio promedio durante el periodo 2017-2020 como se detalla n Figura 4, mientras que el 30\% vende a un precio de $\$ 225$ debido a factores como la venta a intermediarios, el $26 \%$ comercializa el quintal de café a $\$ 240$ dólares que les permite generar un ingreso para su economía de subsistencia.

\section{Figura 4}

Precio Promedio del quintal de café durante el periodo 2017 al 2020

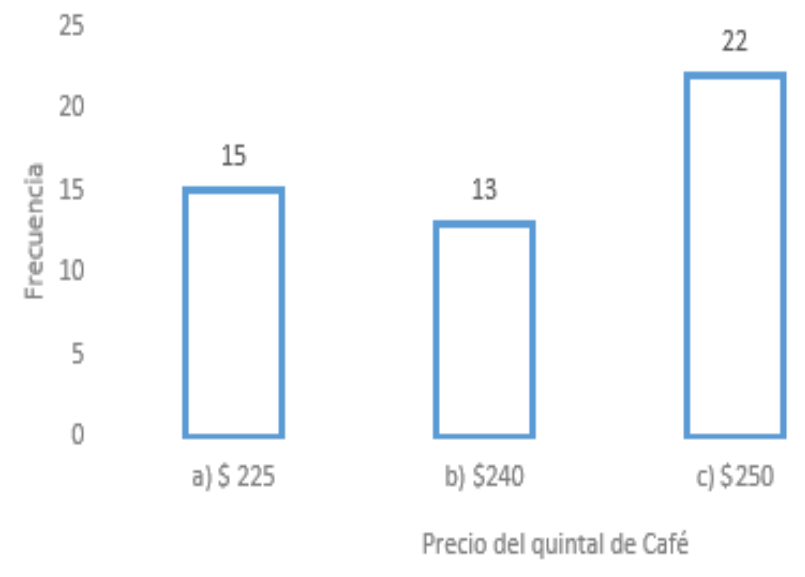

La provincia de El Oro se caracteriza por su diversidad en el mercado, los canales de distribución de la producción de realiza de diferentes formas, por medio del método de la encuesta utilizada se establece que el $44 \%$ se realiza de forma directa entre el productor y consumidor final, seguido del $34 \%$ que indican que comercializan con intermediarios para poder vender su producto debido a que son pequeños productores, por último se ubica la venta al mayorista que están representados por el 22\% generalmente son los productores que tiene mayor a 10 hectáreas en producción.

\section{Figura 5}

Canales de comercialización de los productores de café

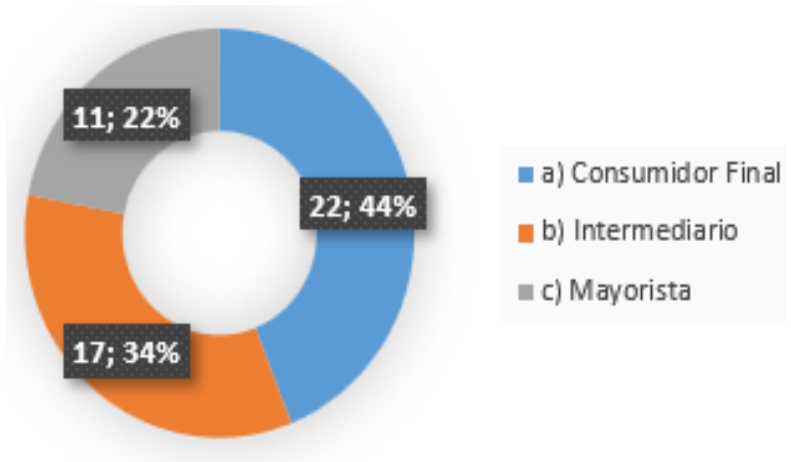


La agricultura campesina no es sólo una pequeña agricultura sino una agricultura difícil. Esto significa que el grado de riesgo para el productor es mayor. Por esta razón y por la inestabilidad en los precios del mercado, así como por su pobreza, las familias campesinas tienen un comportamiento económico caracterizado por una gran aversión al riesgo, tal como lo muestra en la Figura 6, el 44\% de los productores ingresos por ventas de café anuales de $\$ 500$ a $\$ 1000$, mientras que el $30 \%$ de los productores de café de $\$ 1000$ a \$1500 dólares producto de sus ventas, con el $26 \%$ de los productores de café perciben ingresos mayores a los $\$ 1500$ dólares que están dentro del grupo de los grandes productores.

\section{Figura 6}

\section{Ingresos anuales por la venta de café}

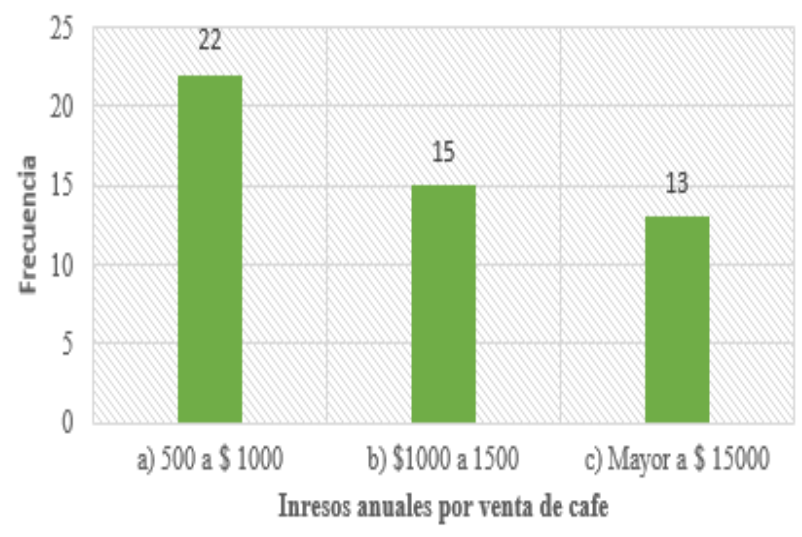

Los pequeños, medianos y grandes productores de café que hacen vida en el cantón Zaruma, son de vital importancia para el dinamismo de la economía agrícola mediante su comercialización del producto, obteniendo ingresos dignos para mejorar su calidad de vida, la de sus familias (Pincay \& Unda, 2020).

La agricultura familiar campesina (AFC) es responsable en gran magnitud del dinamismo de la economía interna, y su vez un importante componente de la generación de empleo(Belduma \& Barrezueta, 2018). En otro contexto, este sector soporta importantes limitaciones que merman sus capacidades, sobre todo en el acceso a nuevas tecnología, deficiente mano de obra joven y el ineficiente uso y gestión del suelo agropecuario (Osorio \& Pombo, 2019).
De acuerdo a Ocampo \& Alvarez. (2017), para permanecer el tiempo los productores de café, deben de adaptarse a la reorganización social y espacial de los productores donde se desplaza el interés de un eje predominantemente agrario hacia otro en el cual se incorporan aspectos ambientales, de salud, de usos generales o alternativos de los espacios rurales, representan el marco para el desarrollo de unas relaciones sociales de producción destinadas a maximizar la comercialización y aportar dividendos altamente provechosos para las familias campesinas rurales.

Según Méndez et al. (2014)en la provincia de Manabí (Ecuador en su estudio sobre caracterización de fincas cafetaleras en la localidad de Jipijapa (Manabí, Ecuador) menciona que la superficie que son consideras pequeñas al compararse con plantaciones en la provincias, donde la mayor proporción de caficultores poseen fincas $>10$ ha, es decir que el cantón Zaruma se agrupan en su mayoría pequeños productores, esta cifra no es exacta, ya que no existe estadística que indique el número total de familias productoras de café.

\section{Conclusiones}

Con base en los resultados obtenidos se concluye que en general, que las tres cuartas partes de los productores de café en el cantón Zaruma oscilan en un rango de 55 a 60 años de edad, además las unidades productivas están lideradas en un $70 \%$ por el género masculino. Más de la mitad de los productores de café están en manos de pequeños productores con rango de 1 a 5 ha con son la base del dinamismo económico local.

Dado este contexto el $44 \%$ de los productores venden a $\$ 250$ dólares el quintal de café, logrando obtener ingreso promedio mayor a \$1500 por hectárea al año los que pertenecen a la asociación, mientras que los demás venden a los intermediarios, la actividad cafetera para la mayoría de campesinos permite elevar su calidad de vida y a su vez generan empleo de forma directa e indirecta. 
Los resultados obtenidos en este trabajo coinciden con lo establecido en nuestro objetivo, siendo la importancia del café como un producto agrícola comercializado en el mercado cantonal y provincial. Se logro demostrar que la economía de las personas que se dedican a este tipo de actividad si les resulta rentable para cubrir sus necesidades.

Otro factor de gran importancia es que el precio y las condiciones climatológicas para producir el café; inciden directamente en las ganancias, por lo tanto; si existe un precio bajo o un mal clima las ganancias para los agricultores serán muy bajas y no lograrán cubrir ni los costos de producción.

\section{Referencias bibliográficas}

Asociación Nacional de Exportadores de Café. (2020). Precios y exportaciones de café. https://www.anecafe.org.ec/

Banco Central del Ecuador. (2019). Reporte de coyuntura económica del sector agropecuario. In Boletín COFIDE (Issue 93). www.bce.ec

Belduma, R., \& Barrezueta, S. (2018). Migración de agricultores del Cantón Chilla: un estudio de caso desde la preceptiva social y económica. Revista Científica Agroecosistemas, 89. https://aes.ucf.edu. cu/index.php/aes/article/view/224/250

Chango, M. A. (2021). Visor Redalyc Análisis de la competitividad de las exportaciones de café de Ecuador versus Colombia y Brasil hacia el mercado de USA. X-Pedientes Económicos, 6, 65-82. http://portal.amelica.org/ameli/ jatsRepo/392/3922449005/index.html

Figueroa, E., Pérez, F., \& Godínez, L. (2019). Los precios de café en la producción y las exportaciones a nivel mundial. Revista Mexicana de Economía y Finanzas, 14(1), 41-56. https://doi.org/10.21919/REMEF. V14I1.358

García, J. M., \& Quezada, A. del C. (2021).
La asociatividad, sustentabilidad y certificaciones en la producción cafetalera enelsurdelEcuador.EconomíaCoyuntural, 6, 33-59. http://www.scielo.org.bo/scielo. php? script $=$ sci_arttext\&pid $=\mathrm{S} 2415$ $06222021000200004 \& \operatorname{lng}=$ es\&nrm $=$ iso

Instituto Nacional de Estadística y Censos. (2019). Encuesta de Superficie y Producción Agropecuaria Continua (ESPAC) 2018. https://www.ecuadorencifras.gob.ec/ documentos/web-inec/Estadisticas agropecuarias/espac/espac-2018/ Presentacion de principales resultados.pdf

López, J., Quezada, R., \& Castillo, M. del C. (2017). Migración interna y urbanización sin eficiencia en países en desarrollo: evidencia para Ecuador. Papeles de Población. https://www.redalyc.org/ articulo.oa? $\mathrm{id}=11254687004$

Márquez, L. E., Cuétara, L. M., Cartay, R. C., \& Ferrer, N. J. L. (2019). Desarrollo y crecimiento económico: Análisis teórico desde un enfoque cuantitativo. Revista de Ciencias Sociales, 1, 233-253. https://www.redalyc.org/ journal $/ 280 / 28063104020 / \mathrm{html} /$

Méndez, M., Otiniano, A., Ventura, R., \& Hidalgo, O. (2014). Caracterización de fincas cafetaleras en la localidad de Jipijapa (Manabí, Ecuador). Ecología Aplicada, 13(2), 187-192. http://www.scielo.org.pe/scielo. php? script $=$ sci_arttext\&pid $=$ S 1726 $22162014000200013 \& \operatorname{lng}=$ es\&nrm=iso \&tlng=es

Ocampo, O. L., \& Alvarez, L. M. (2017). Tendencia de la producción y el consumo del café en Colombia. Apuntes Del Cenes, 36(64), 139-165. https:// doi.org/10.19053/01203053.v36. n64.2017.5419

Osorio, N. Y., \& Pombo, O. A. (2019). La evolución tecnológica y la percepción de la calidad ambiental de los caficultores 
de El Águila, Valle del Cauca, Colombia. Frontera Norte, 31(0), 0. https://doi. org/10.33679/RFN.V1I1.1988

Pincay, C. V. C., \& Unda, S. B. (2020). Aspectos sociales y económicos: caso productores de café en la provincia El Oro. Revista Metropolitana de Ciencias Aplicadas, 3(1), 71-75. https://remca.umet.edu.ec/ index.php/REMCA/article/view/233

Ponce, L. A., Orellana, K. D., \& Acuña, I. R. (2018). Situación de la caficultura ecuatoriana: perspectivas. Revista Estudios Del Desarrollo Social: Cuba y América Latina, 6, 307-325. http://scielo. sld.cu/scielo.php?script $=$ sci_arttext\&pid $=\mathrm{S} 2308-01322018000100015$

Vaca, A. P., Suarez, D. O., \& Velásquez, R. A. (2018). Situación de la caficultura ecuatoriana: perspectivas. Revista Estudios Del Desarrollo Social: Cuba y América Latina, 6(1), 307$325 . \quad \mathrm{http}: / /$ scielo.sld.cu/scielo. php?script $=$ sci_arttext\&pid $=$ S2308$01322018000100015 \& \operatorname{lng}=\mathrm{es} \& \mathrm{nrm}=\mathrm{iso}$ \&tlng=es

Valdez, M., \& Vizcaya, Z. (2009). Análisis de las relaciones socioproductivas de caficultores de los municipios Morán y Andrés Eloy Blanco del estado Lara, Venezuela. Bioagro, 21(2), 111-116. http://ve.scielo.org/scielo. php? script $=$ sci_arttext\&pid $=\mathrm{S} 1316$ $33612009000200005 \& \operatorname{lng}=\mathrm{es} \& \mathrm{nrm}=\mathrm{iso}$ \&tlng=es

Bello, G. A. (2016). "PRODUCCIÓN $Y$ EXPORTACIÓN DE CAFÉ ZARUMA TIPO ARÁBIGO DE LA HACIENDA "EL TABLÓN" HACIA EL MERCADO ESPAÑOL”. Quito. Obtenido de 